\title{
STEADY STATE STABILITY ANALYSIS AND ENHANCEMENT OF THREE MACHINE NINE BUS POWER SYSTEM USING TCPS FACTS DEVICE
}

\author{
Divya Rishi Shrivastava ${ }^{1}$, Daneshwari R Akkishetti ${ }^{2}$ \\ ${ }^{1}$ Assistant Professor, Electrical \& Electronics Dept., Manipal University Jaipur, Rajasthan, India \\ ${ }^{2}$ Lecturer, Electrical \& Electronics Dept., Jain College of Engineering Belgaum, Karnataka, India
}

\begin{abstract}
Power System stability study is the important parameter of economic, reliable and secure system planning and operation. Studies are important during the planning and conceptual design stages of the project as well as during the operating life of the plant periodically. This paper presents the power system steady state stability analysis for IEEE- 9 bus test system and examines influence of TCPS FACTS device based controller on test system. It is assumed that system under study has been perturbed from a steady state equilibrium that prevailed prior to the application of the disturbance. If system is stable, we would expect that for temporary or permanent disturbance, system will acquire initial or new operating state after a transient period. The stability study is accessed using Lyapunov's first method. The effectiveness of damping controller in enhancing the steady state stability is investigated by incorporating available constraints. For analysis MATLAB software is employed. The conclusions have been drawn here, based on theoretical and mathematical analysis so as to provide an insight and better understanding of steady state stability of considered multi machine power system.
\end{abstract}

Key Words: Lyapunov's first method, Steady-state stability, Phase portrait, FACTS device, supplementary modulation controller, eigen value, synchronizing power coefficient, IEEE-9 Bus Test System, Load Flow Study, Differential algebraic equation.

\section{INTRODUCTION}

Power system stability may be defined as property of a power system that enables it to remain in a state of operating equilibrium under normal operating conditions and to regain an acceptable state of equilibrium after being subjected to a disturbance [4]. The oscillations, which are typically in the frequency range of 0.2 to $3.0 \mathrm{~Hz}$, might be excited by the disturbances in the system [1][13] or, in some cases, might even build up spontaneously. A power system at a given operating condition may be unstable due to large disturbance; still such a system can be operated, though insecurely. However, if the system is small signal unstable at a given operating condition, it cannot be operated. Therefore, steady state stability is a fundamental requirement for satisfactory operation of power system. Instability that may result can be of two forms i)increase in rotor angle through a non-oscillatory or aperiodic mode due to lack of synchronizing torque, or ii)rotor oscillation of increasing amplitude due to lack of sufficient damping torque[13].With the advent of FACTS devices, and due to recent advances in power electronics, its capability to enhance oscillation damping and system stability has been explored as potential application. With advent of high power, high speed electronic FACTS controllers have been mainly used for solving various power system control problems such as voltage regulation, power flow, and transfer capability enhancement. As supplementary functions, they have been in use for damping the inter area modes and enhancing power system stability [3].

This paper studies theoretical and mathematical analysis of IEEE 3- machine - 9 bus power system and the influence of the TCPS FACTS device based controller on the steady state stability about an operating condition. Here, controller parameters are obtained by incorporating available constraints. The Mathematical analysis is carried out in MATLAB using eigenvalue analysis, phase portrait and time domain analysis of state variable of power system when subjected to small disturbance.

\subsection{IEEE -3 Machine 9 Bus Power System}

We have considered the popular Western System Coordinated Council (WSSC), IEEE 3-machine, 9-bus system shown in fig.1. This system is also appearing in references [8] and [10].The base MVA is 100 and system frequency is $60 \mathrm{~Hz}$. The system data are given in Appendix I. Classical model of the generators are employed for analysis. The operating conditions are obtained by using Load flow analysis. Eigen value analysis and phase portrait for concerned state variable are drawn about available operating state. The Enhancement in steady state stability is analyzed by incorporating TCPS FACTS device based controller, the gains of which are taken by incorporating available constraints. For Analysis TCPS FACTS device is placed between Machine 1 and 2. 


\subsection{Mathematical Model}

A load flow study of a power system shown in Fig. 1, is done to determine electrical power output of machine $i$ and to calculate values of $E_{\mathrm{i}} \angle \delta_{0}$ for all generators.

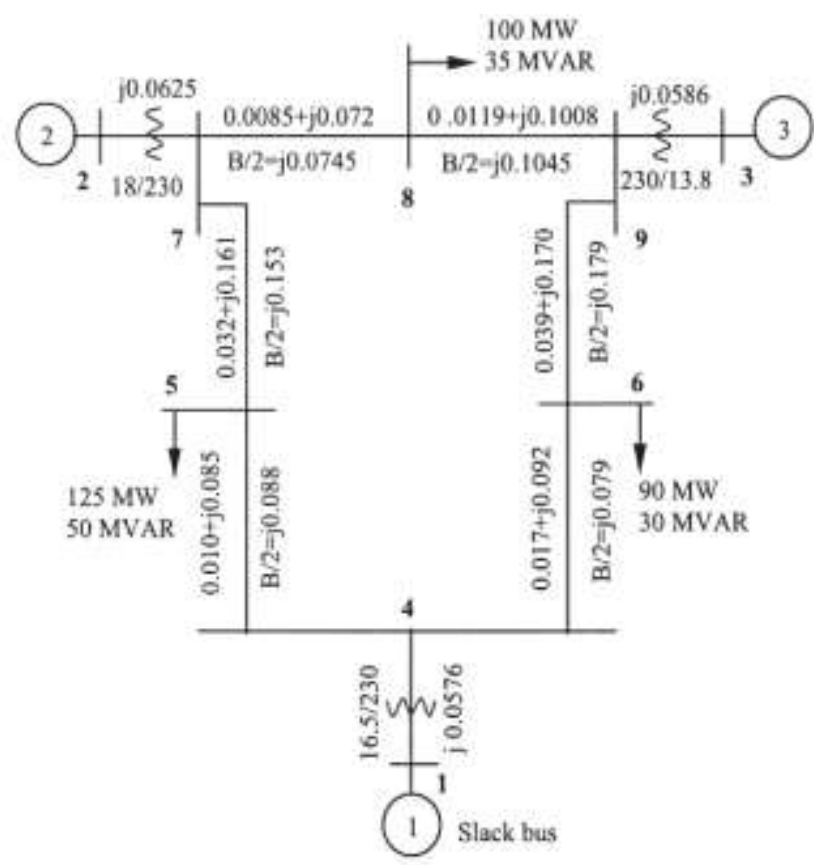

Fig-1 IEEE 3-machine 9-bus power system

The power into the network at node $\mathrm{i}$,

$P_{\theta_{\mathrm{i}}}=E_{\mathrm{i}}^{2} G_{\mathrm{ii}}+\sum_{\substack{j=1 \\ j \neq \mathrm{i}}}^{n} E_{\mathrm{i}} E_{j} Y_{\mathrm{i} j} \cos \left(\theta_{\mathrm{i} j}-\delta_{\mathrm{i}}+\delta_{j}\right) i=1,2,3 \ldots n$

Where,

$\bar{Y}_{i j}=Y_{i j} \angle \theta_{i j}=G_{i j}+j B_{i j}$

$=$ negative of the transfer admittance between nodes $\mathrm{i}$ and $\mathrm{j}$.

$\bar{Y}_{\mathrm{ii}}=Y_{\mathrm{ii}} \angle \theta_{\mathrm{ii}}$

$=$ driving point admittance for node $\mathrm{i}$.

Once Y matrix is calculated, we eliminate all the nodes expect for the internal generator nodes and obtain the $\mathrm{Y}$ matrix for reduced network. The reduction can be achieved by matrix operation considering fact that all the nodes have zero injection currents except for the internal generator nodes. In a power system with $\mathrm{n}$ generators, the nodal equation can be written as,

$\left[\begin{array}{c}I_{n} \\ 0\end{array}\right]=\left[\begin{array}{ll}Y_{n n} & Y_{n Y} \\ Y_{n n} & Y_{r y}\end{array}\right]\left[\begin{array}{l}V_{n} \\ V_{r}\end{array}\right]$

Where subscript $\mathrm{n}$ is used to denote generator nodes and subscript $r$ is used for the remaining nodes.

Expanding Eq. (2)

$I_{n}=Y_{n n} V_{n}+Y_{n Y} V_{Y}$

$$
0=Y_{r n} V_{n}+Y_{r y} V_{r}
$$

From which we eliminate $V_{r}$ to find

$$
\begin{gathered}
I_{n}=\left(Y_{n n}-Y_{n Y} Y_{r y}{ }^{-1} Y_{r n}\right) V_{r} \\
Y_{r e d}=\left(Y_{n n}-Y_{n Y} Y_{r Y}^{-1} Y_{r n}\right)
\end{gathered}
$$

It has dimensions $(n \times n)$ where $\mathrm{n}$ is the number of generators. This convenient analytic technique that can be used only when the loads are treated as constant impedance [8][11]. The equation of motion for $i$ th machine given by,

$$
\begin{aligned}
& \frac{2 H_{i}^{i}}{\omega_{r}} \frac{d \omega_{i}^{i}}{d t}=P_{m i}-\left[E_{i}^{2} G_{i i}+\sum_{\substack{j=1 \\
j \neq i}}^{n} E_{i} E_{j} Y_{i j} \cos \left(\theta_{i j}-\delta_{i}+\delta_{j}\right)\right] \\
& \frac{\mathrm{d} \delta_{i}}{\mathrm{dt}}=\omega_{\mathrm{i}}-\omega_{\mathrm{r}}
\end{aligned}
$$

The electrical power $P_{\text {ei }}$ is given by Eq.(1). Linearizing Eq.(1) and (5) about initial operating condition [4] represented by $\delta=\delta_{0}$ yields,

$\Delta P_{e}=\frac{\partial P_{e}}{\partial \delta} \Delta \delta$

$k_{\text {sij }}=E_{i} E_{j} Y_{i j} \sin \left(\theta_{i j}-\delta_{i}+\delta_{j j}\right) \Delta \delta$

defined as synchronizing power coefficient [4].

The test power system is now linearized about initial operating condition available from load flow. The classical model [6][7] of a synchronous machine is used to study the stability of a power system. Linearizing multi machine system about an initial operating condition using equations (4) and (5) gives,

$$
\begin{aligned}
& \frac{d^{2} \Delta \delta_{1}}{d t^{2}}=\frac{\omega_{y}}{2 H_{1}} \Delta P_{\mathrm{m} 1}-\frac{\omega_{y}}{2 H_{1}}\left[k_{g 12} \Delta \delta_{12}+k_{g 1 a} \Delta \delta_{1 a}\right] \\
& \frac{d^{2} \Delta \delta_{2}}{d t^{2}}=\frac{\omega_{y}}{2 H_{2}} \Delta P_{\mathrm{m} 2}-\frac{\omega_{y}}{2 H_{2}}\left[k_{g 2 a} \Delta \delta_{2 a}+k_{g 21} \Delta \delta_{21}\right] \\
& \frac{d^{2} \Delta \delta_{a}}{d t^{2}}=\frac{\omega_{y}}{2 H_{a}} \Delta P_{\mathrm{ma}}-\frac{\omega_{y}}{2 H_{a}}\left[k_{g a 1} \Delta \delta_{\mathrm{a} 1}+k_{\mathrm{ga} 2} \Delta \delta_{\mathrm{a} 2}\right]
\end{aligned}
$$

When generator 2 and 3 are swinging with respect to generator 1 ,

$$
\begin{aligned}
& \frac{d}{d t} \omega_{21}=\left[\frac{\omega_{y}}{2 H_{2}} \Delta P_{\mathrm{m} 2}-\frac{\omega_{y}}{2 H_{1}} \Delta P_{\mathrm{m} 1}\right] \\
& -\left[\frac{\omega_{y}}{2 H_{2}} k_{g 12}+\frac{\omega_{y}}{2 H_{2}} k_{g 2 a}+\frac{\omega_{y}}{2 H_{2}} k_{g 21}\right] \Delta \delta_{21} \\
& +\left[\frac{\omega_{y}}{2 H_{2}} k_{g 2 a}-\frac{\omega_{y}}{2 H_{1}} k_{g 1 a}\right] \Delta \delta_{31}
\end{aligned}
$$


$\frac{d}{d t} \omega_{\mathrm{a} 1}=\left[\frac{\omega_{y}}{2 H_{a}} \Delta P_{\mathrm{ma}}-\frac{\omega_{r}}{2 H_{1}} \Delta P_{\mathrm{m} 1}\right]$

$-\left[\frac{\omega_{y}}{2 H_{a}} k_{g a 1}+\frac{\omega_{y}}{2 H_{1}} k_{g 1 a}+\frac{\omega_{y}}{2 H_{a}} k_{g a 2}\right] \Delta \delta_{a 1}$

$+\left[\frac{\omega_{y}}{2 H_{3}} k_{g 32}-\frac{\omega_{y}}{2 H_{1}} k_{g 12}\right] \Delta \delta_{21}$

From Eq. (8) the state space representation of the test system is given by,

$\frac{d}{d t}\left[\begin{array}{l}\Delta \delta_{21} \\ \Delta \omega_{21} \\ \Delta \delta_{31} \\ \Delta \omega_{31}\end{array}\right]=\left[\begin{array}{cccc}0 & 1 & 0 & 0 \\ A_{21} & 0 & A_{23} & 0 \\ 0 & 0 & 0 & 1 \\ A_{41} & 0 & A_{43} & 0\end{array}\right]\left[\begin{array}{l}\Delta \delta_{21} \\ \Delta \omega_{21} \\ \Delta \delta_{31} \\ \Delta \omega_{31}\end{array}\right]+\left[\begin{array}{c}0 \\ -w_{r} / 2 H_{1} \\ 0 \\ -w_{r} / 2 H_{1}\end{array}\right] \Delta P m_{1}+$ $\left[\begin{array}{c}0 \\ w_{r} / 2 H_{2} \\ 0 \\ 0\end{array}\right] \Delta P m_{2}+\left[\begin{array}{c}0 \\ 0 \\ w_{r} / 2 H_{3} \\ 0\end{array}\right] \Delta P m_{3}$

Where $A_{i j}$ Eq. are formed using Eq. (8). Eq. (9) is written in standard control theory form $\Delta X=A \Delta X+B \Delta U$, where $\mathrm{X}$ is state variable vector and $\mathrm{U}$ is the control variable vector. Applying controllability criterion to all three input matrix,

$I=\left[B_{\mathrm{i}}: B_{j}: B_{k}\right]$

It should follow,

$\mathrm{C}=\mathrm{n}[1: 1: 1]$

Where $\mathrm{n}$ is size of Jacobian matrix.

\section{TCPS FACTS DEVICE BASED CONTROLLER MODELLING}

Thyristor controlled phase shifter is used to enhance steady state stability of power system. TCPS FACTS device based controller enable fast control of the phase angle ( $\varnothing$ )and it is determined as non linear function of rotor angle and speed. It is used to damp low frequency oscillation. For TCPS FACTS device based damping controller generally Supplementary Modulation Controller are recommended [2], by designing a SMC for the damping controller based on rotor speed signal as input, it is possible to enhance the damping and stabilize swing mode. TCPS FACTS device based controllers are based on conventional lead-lag type. Here $T_{1}$ and $T_{2}$ provide the required phase compensation to give necessary damping. $T_{W}$ is washout circuit time constant, wash out circuit serves as a high pass filter with time constant $T_{W}$ varies from 10 s to $20 \mathrm{~s}$ [2].

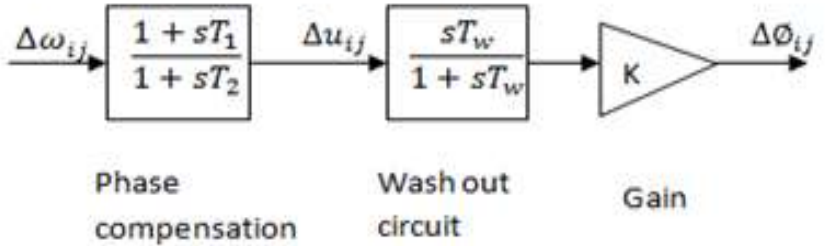

Fig-2 Structure of TCPS based controller

Controllable parameter of the TCPS FACTS device based controller is $\delta$ that is rotor angle of generator and the control law is,

$\frac{d \Delta \emptyset_{i j}}{d t}=\frac{d \Delta \delta_{i j}}{d t}$

The transfer function of controller is given by,

$H(s)=\left[\frac{s T_{W}}{1+s T_{w}}\right] K\left[\frac{1+s T_{1}}{1+s T_{2}}\right]$

Differential algebraic equation of damping controller can be formulated from Eq.(10)

$\Delta u_{i j}^{\circ}=\frac{T_{1}}{T_{2}} \Delta \omega_{i j}-\frac{1}{T_{2}} \Delta u_{i j}+\frac{1}{T_{2}} \Delta \omega_{i j}$

$\Delta \dot{\varphi}_{\mathrm{ij}}=K \Delta \tilde{u}_{\mathrm{ij}}-\frac{\Delta \varphi_{\mathrm{ij}}}{T_{w}}$

Here, controller parameters are obtained by incorporating available constraints such as,

i) No new mode of oscillation should evolve

ii) Damping in the system that should be, $0<\zeta \leq 1$

iii) All states variables should be controllable

2.1 Mathematical Modelling IEEE 9 Bus Power System with TCPS Facts Device

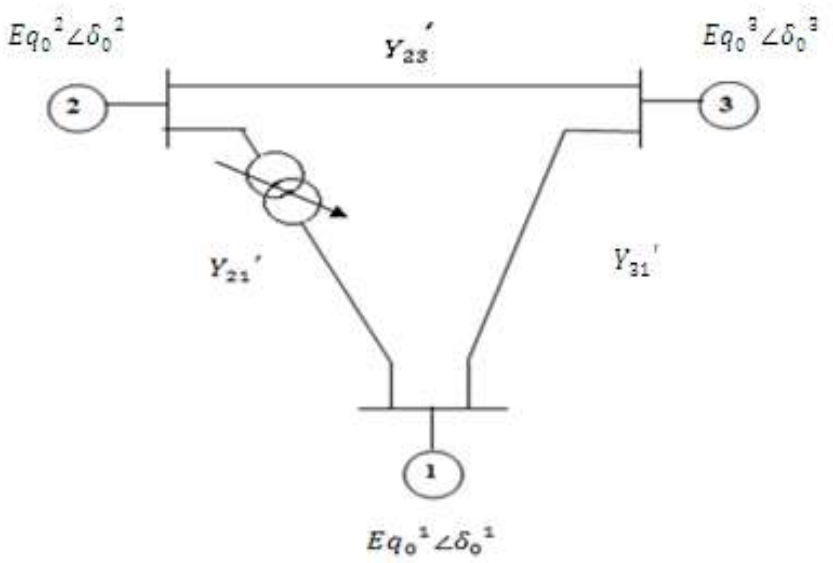

Fig-3 Reduced figure 1 with damping controller

The test power system is equipped with TCPS FACTS device based controller near generator terminal between bus 2 and 1 as shown in Fig.3. As losses of the thyristors are a 
small proportion of the DC link power and quadrature power inserted by transformer is small proportion of the line primary power so that phase shifter losses are neglected [12] and Time constant of thyristors is very short; and can be neglected for computation [12]. The dynamic equation of system with damping controller are obtained by Eq. (8) and (11), linearizing these equations about operating point we get,

$\Delta \tilde{u}_{21}=\frac{1}{T_{2}} \Delta w_{21}-\frac{1}{T_{2}} \Delta u_{21}$

$-\frac{T_{1}}{T_{2}}\left[\frac{\omega_{Y}}{2 H_{2}} k_{g 21}+\frac{\omega_{y}}{2 H_{2}} k_{s 2 a}+\frac{\omega_{Y}}{2 H_{1}} k_{s 12}\right] \Delta \delta_{21}$

$-\frac{T_{1}}{T_{2}}\left[\frac{\omega_{y}}{2 H_{2}}\left[k_{s 21}+k_{s 2 a}\right] \Delta \varphi_{21}+\frac{T_{1}}{T_{2}}\right.$

$\Delta \varphi_{21}=k\left[\Delta \dot{u}_{21}\right]-\frac{1}{T_{W}}\left[\Delta \varphi_{21}\right]$

The state space representation of test system with damping controller is obtained by Eq.(8) and Eq.(12) we get,

$\frac{d}{d t}\left[\begin{array}{l}\Delta \delta_{21} \\ \Delta \omega_{21} \\ \Delta \delta_{31} \\ \Delta \omega_{31} \\ \Delta u_{21} \\ \Delta \phi_{21}\end{array}\right]=\left[\begin{array}{cccccc}0 & 1 & 0 & 0 & 0 & 0 \\ A_{21} & 0 & A_{23} & 0 & 0 & A_{26} \\ 0 & 0 & 0 & 1 & 0 & 0 \\ A_{41} & 0 & A_{43} & 0 & 0 & 0 \\ A_{51} & A_{52} & A_{53} & 0 & A_{55} & A_{56} \\ A_{61} & A_{62} & A_{63} & 0 & A_{65} & A_{66}\end{array}\right]\left[\begin{array}{c}\Delta \delta_{21} \\ \Delta \omega_{21} \\ \Delta \delta_{31} \\ \Delta \omega_{31} \\ \Delta u_{21} \\ \Delta \phi_{21}\end{array}\right]$

$+\left[\begin{array}{c}0 \\ B_{21} \\ 0 \\ B_{41} \\ B_{51} \\ B_{61}\end{array}\right] \Delta P m_{1}+\left[\begin{array}{c}0 \\ C_{21} \\ 0 \\ 0 \\ C_{51} \\ C_{61}\end{array}\right] \Delta P m_{2}+\left[\begin{array}{c}0 \\ 0 \\ 0 \\ D_{41} \\ 0 \\ 0\end{array}\right] \Delta P m_{3}$

Where $A_{\mathrm{i} j}$ and $B_{\mathrm{i} j}, C_{\mathrm{i} j}$ and $D_{\mathrm{i} j}$ are formed using Eq. (8) and Eq.(12),

Applying controllability criterion to all three input matrix, $I=\left[B_{\mathrm{i}}: B_{j}: B_{k}\right]$

It should follow,

$\mathrm{C}=\mathrm{n}[1: 1: 1]$

Where $\mathrm{n}$ is size of new Jacobian matrix.

\section{RESULTS AND DISCUSSION}

Dynamic performance of power system is simulated using MATLAB software package. Control variable parameters are given in Appendix. The power system is analyzed with and without incorporating TCPS FACTS device based controller. Eigen values of power system without and with facts device are shown in Table I and that of damping controller in table II. Phase portrait and time domain simulation is carried out for the operating condition following a $1 \%$ step perturbation in mechanical power of generator 2 and generator 3 . The phase portrait and time domain results are shown from fig. 4.1 to fig 5.8

Table -1: Eigen Values of Power System as per figure 1

\begin{tabular}{|c|c|}
\hline Eigen Value & $\begin{array}{c}\text { Oscillating } \\
\text { frequency }(\mathrm{Hz})\end{array}$ \\
\hline $0.0000 \pm 13.3597 \mathrm{i}$ & 2.1263 \\
\hline $0.0000 \pm 8.6892 \mathrm{i}$ & 1.3829 \\
\hline
\end{tabular}

As seen in table 1 , that the system is purely oscillatory as there is no damping in the system.

With Step disturbance of $1 \%$ in turbine side of generator 2 is made as per figure 1 , the phase portrait and time domain results so obtained are,

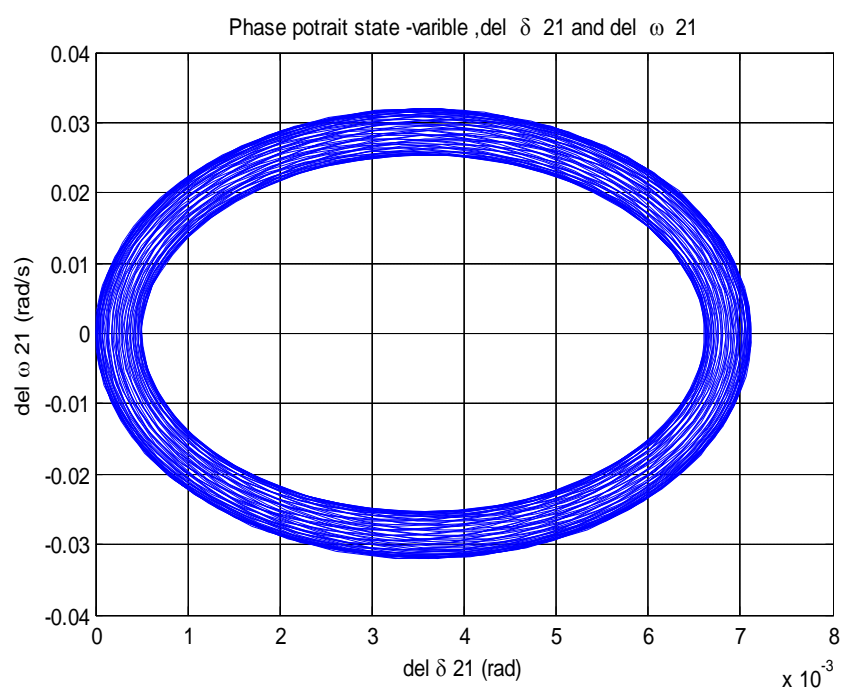

Fig-4.1 Phase portrait state variable, $\Delta \delta_{21}$ and , $\Delta \omega_{21}$

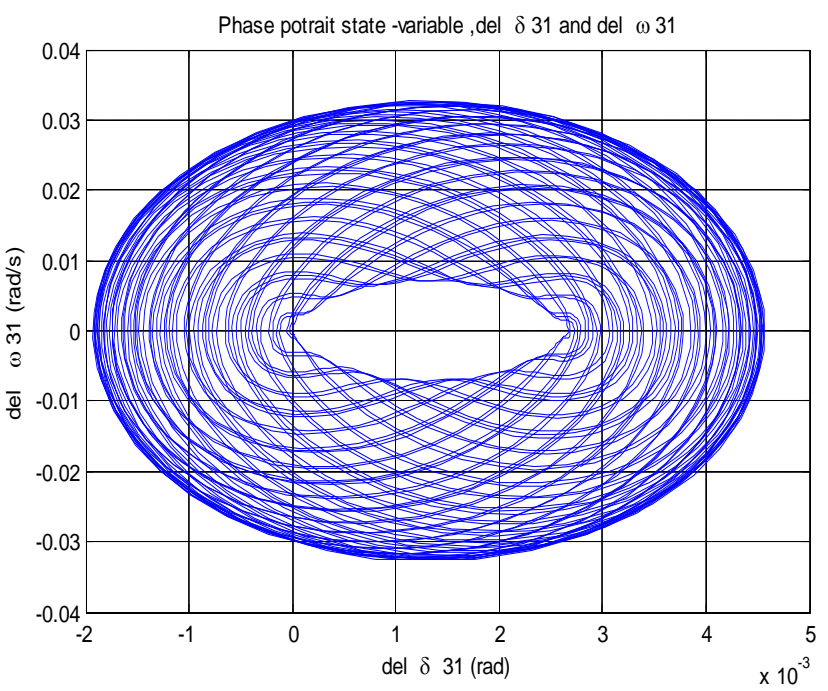

Fig-4.2 Phase portrait state variable, $\Delta \delta_{31}$ and , $\Delta \omega_{31}$ 


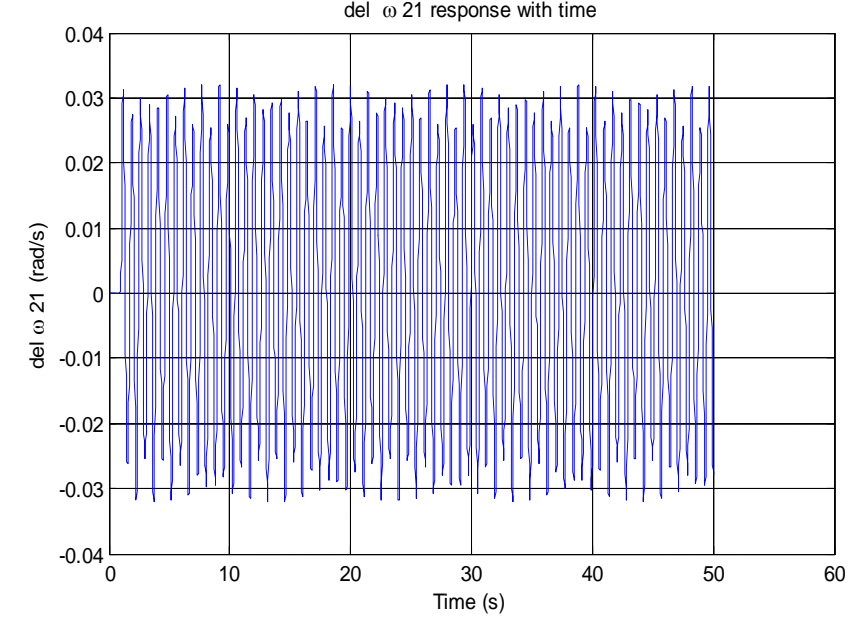

Fig-4.3 Response of $\Delta \omega_{21}$ with time

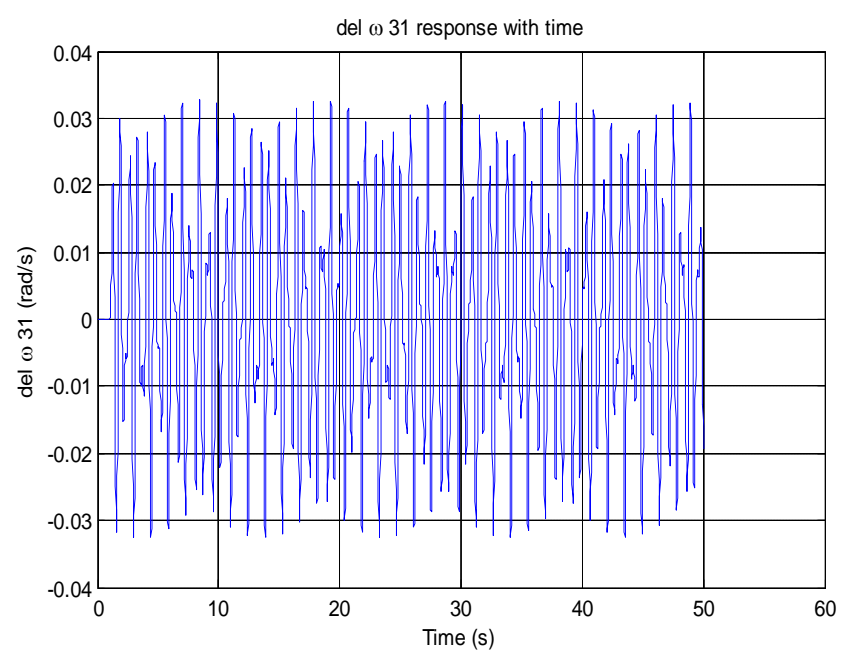

Fig-4.4 Response of $\Delta \omega_{21}$ with time

With Step disturbance of $1 \%$ in turbine side of generator 3 is made as per figure 1 , the phase portrait and time domain results so obtained are,

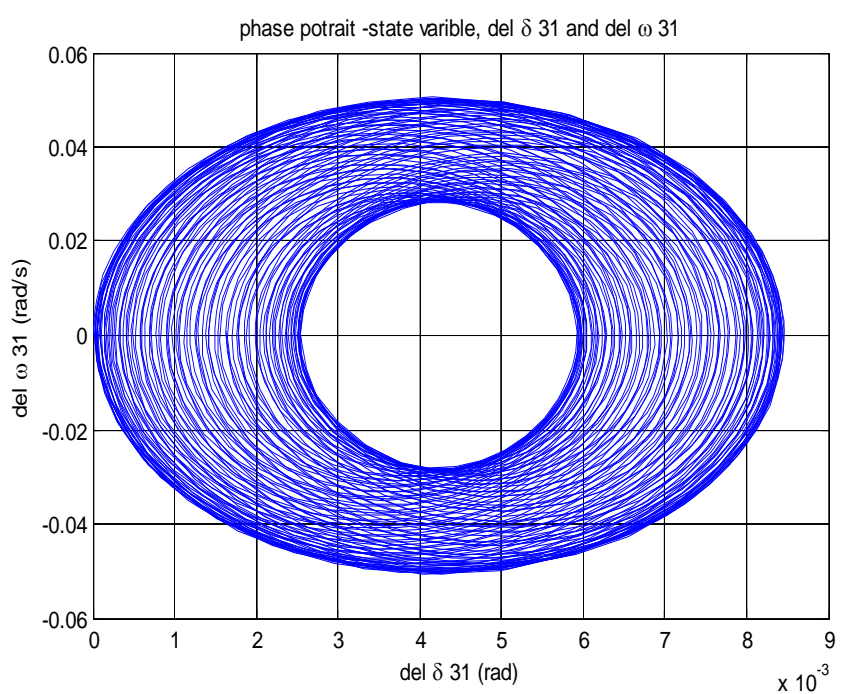

Fig-4.5 Phase portrait state variable, $\Delta \delta_{31}$ and $\Delta \omega_{31}$

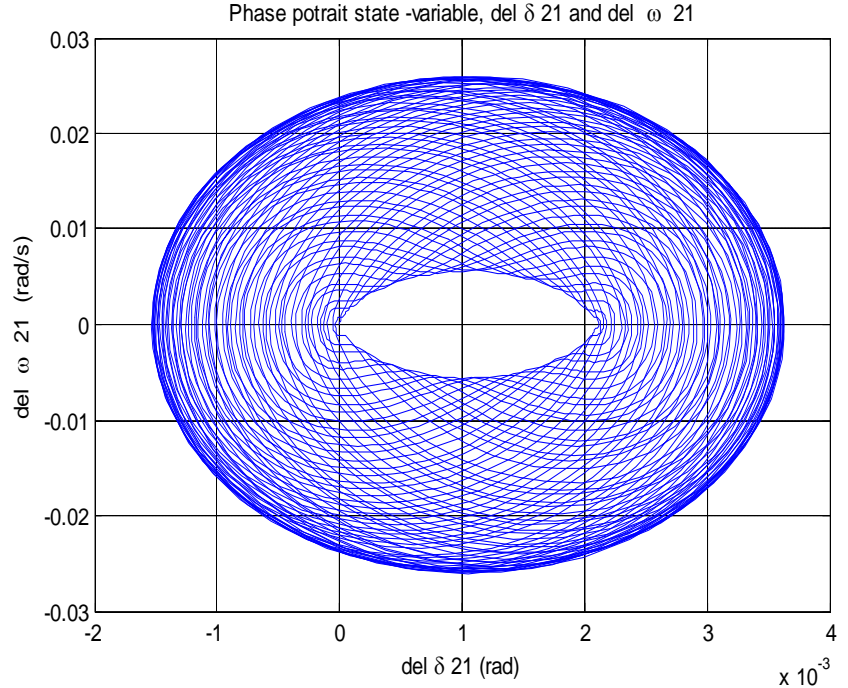

Fig-4.6 Phase portrait state variable, $\Delta \delta_{21}$ and, $\Delta \omega_{21}$

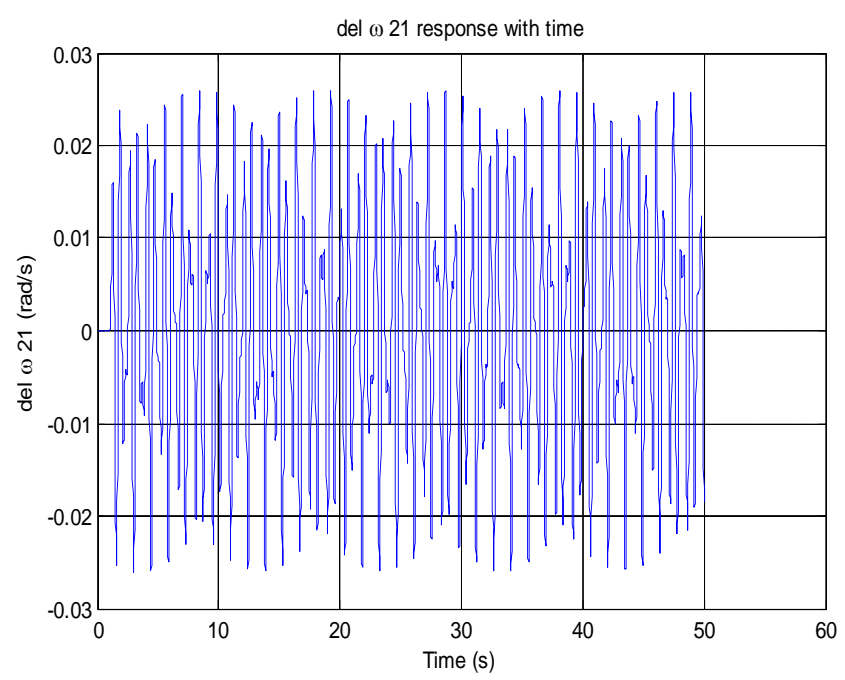

Fig-4.7 Response of $\Delta \omega_{21}$ with time

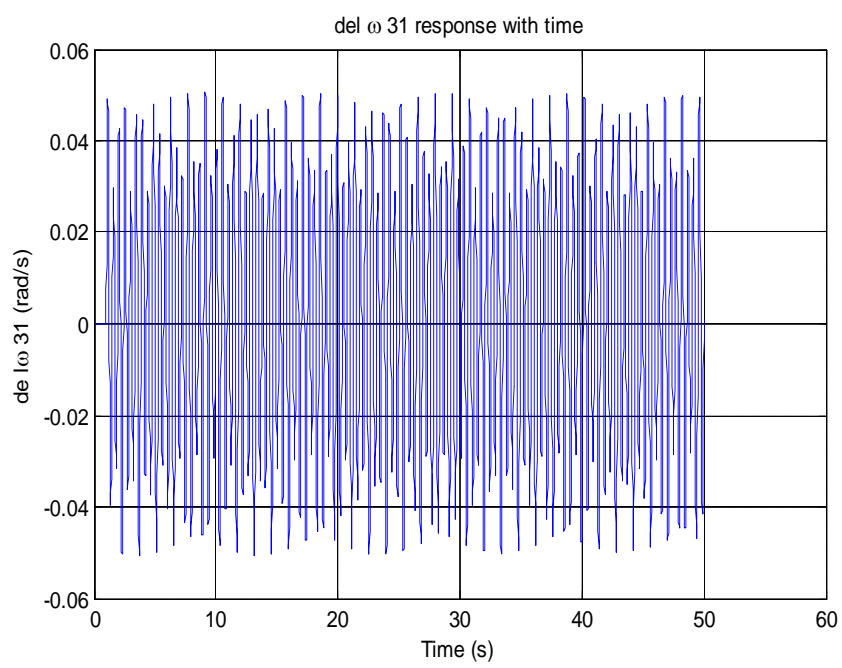

Fig-4.8 Response of $\Delta \omega_{31}$ with time

It is clearly seen in figure 4.1 through 4.8 , that as soon as disturbance is created the system is unable to find new equilibrium state due to lack of damping component. The 
same can also be inferred from different responses of state variables with time.

Through section 2 and 2.1, the eigen values were computed and can be seen in table 2 .

Table -2: Eigen Values of Power System as per figure 3

\begin{tabular}{|c|c|c|}
\hline Eigen value & $\begin{array}{c}\text { Oscillating } \\
\text { frequency }(\mathrm{Hz})\end{array}$ & Zeta $(\zeta)$ \\
\hline-48.6728 & 0 & 1 \\
\hline$-.03281 \pm 13.0505 \mathrm{i}$ & 2.0771 & 0.0251 \\
\hline$-3.6319 \pm 8.2018 \mathrm{i}$ & 1.3054 & 0.4049 \\
\hline-0.1010 & 0 & 1 \\
\hline
\end{tabular}

As seen in table 2, that the system is now damped with zeta lying within o and 1, and also it is to seen that the frequency of oscillation has not changed much from previous value.

With Step disturbance of $1 \%$ in turbine side of generator 2 is made as per figure 3 , the phase portrait and time domain results so obtained are,

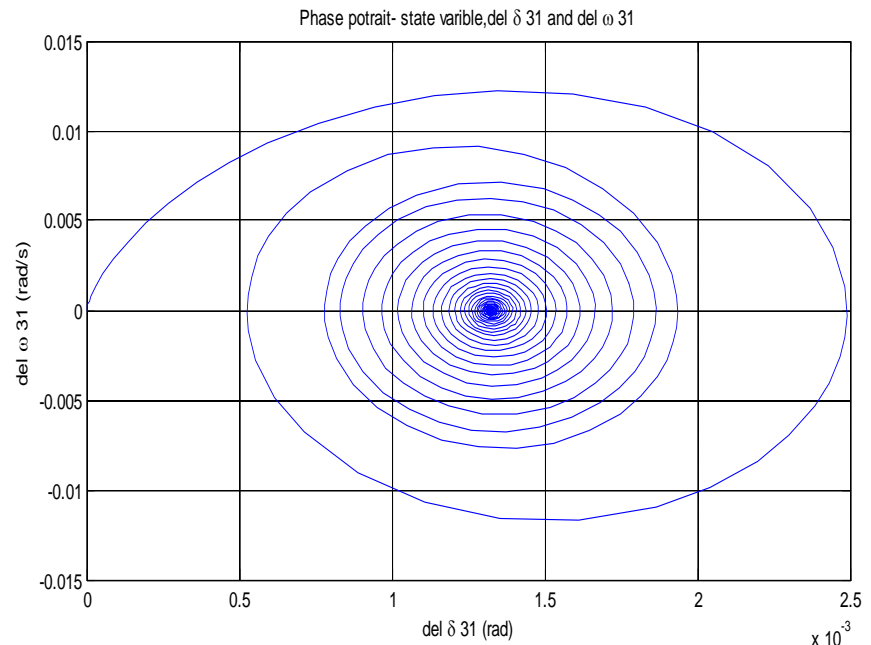

Fig-5.1 Phase portrait state variable, $\Delta \delta_{31}$ and $\Delta \omega_{31}$

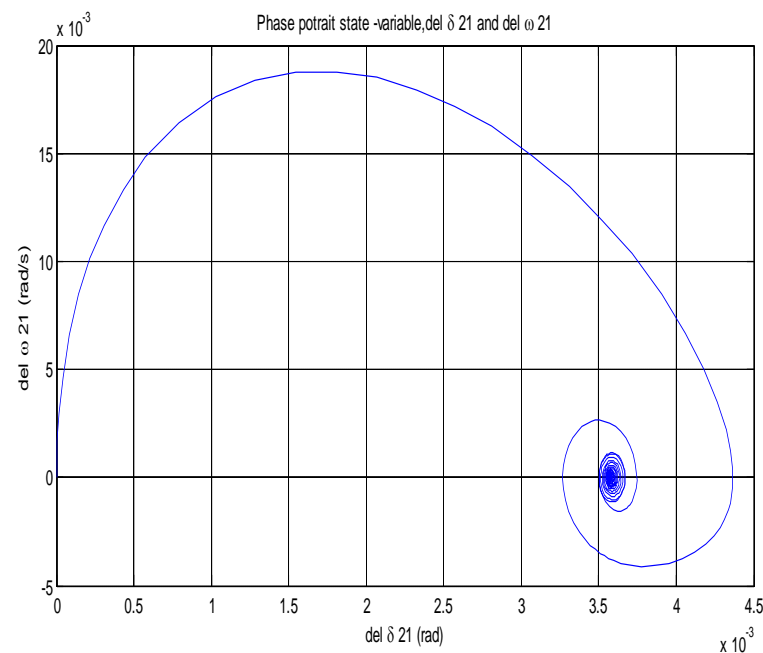

Fig-5.2 Phase portrait state variable, $\Delta \delta_{21}$ and $\Delta \omega_{21}$

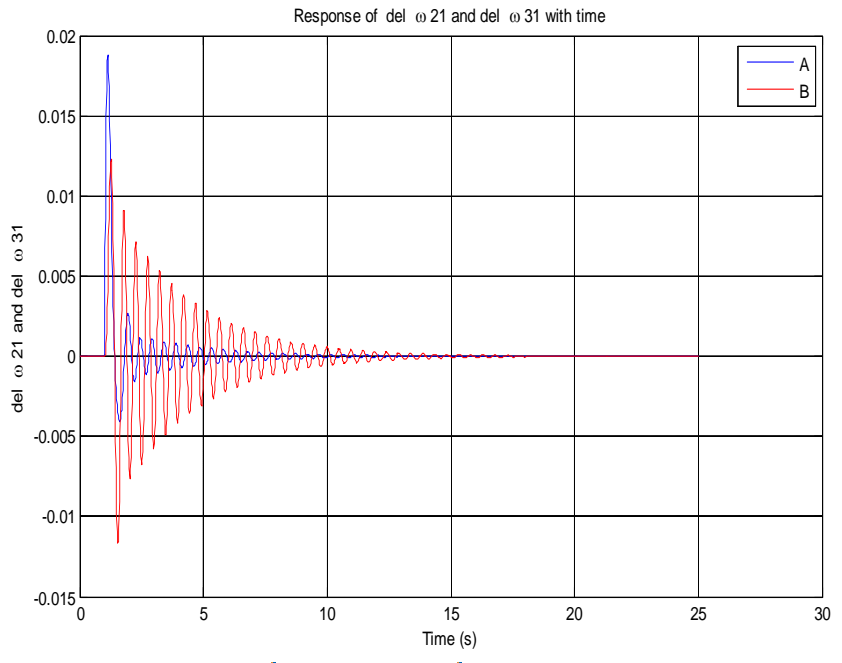

$\mathrm{A}=\Delta \omega_{21}$ and $\mathrm{B}=\Delta \omega_{21}(\mathrm{rad} / \mathrm{s})$

Fig-5.3 Response of $\Delta \omega_{21}$ and $\Delta \omega_{31}$ with time

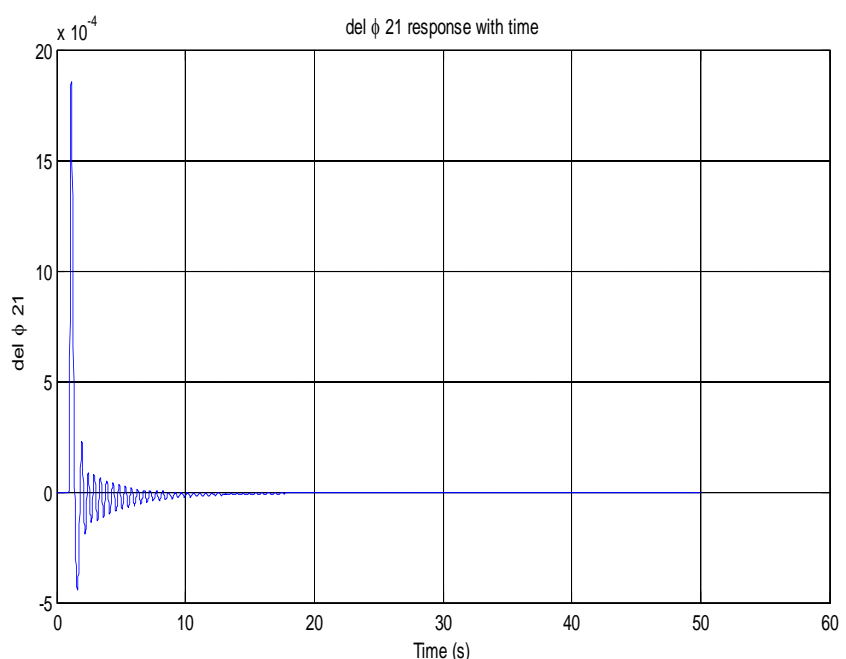

Fig-5.4 Response of $\Delta \Phi_{21}$ with time

With Step disturbance of $1 \%$ in turbine side of generator 3 is made as per figure 3 , the phase portrait and time domain results so obtained are,

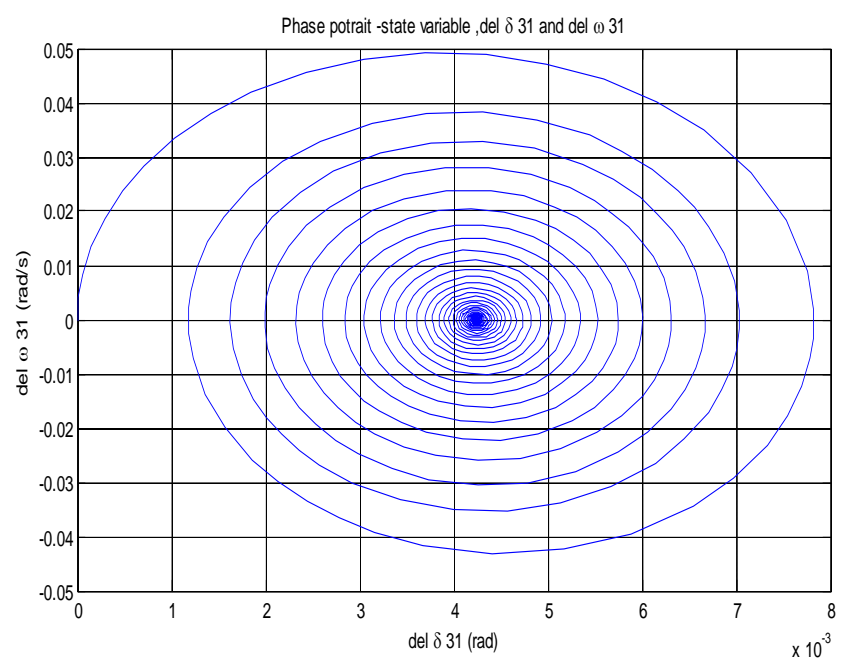

Fig-5.5 Phase portrait state variable, $\Delta \delta_{31}$ and $\Delta \omega_{31}$ 


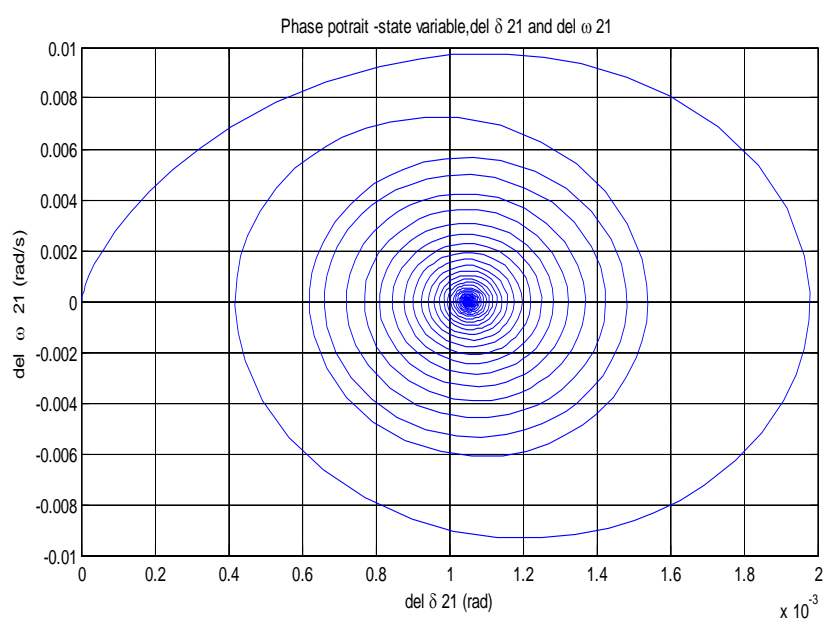

Fig-5.6 Phase portrait state variable, $\Delta \delta_{21}$ and $\Delta \omega_{21}$

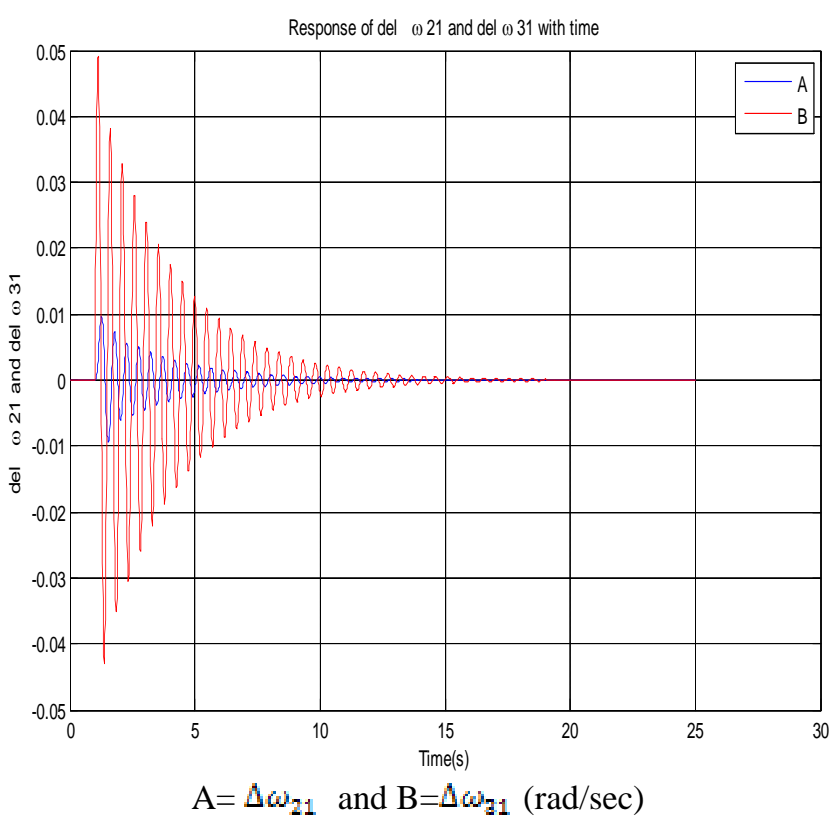

Fig-5.7 Response of $\Delta \omega_{21}$ and $\Delta \omega_{31}$ with time

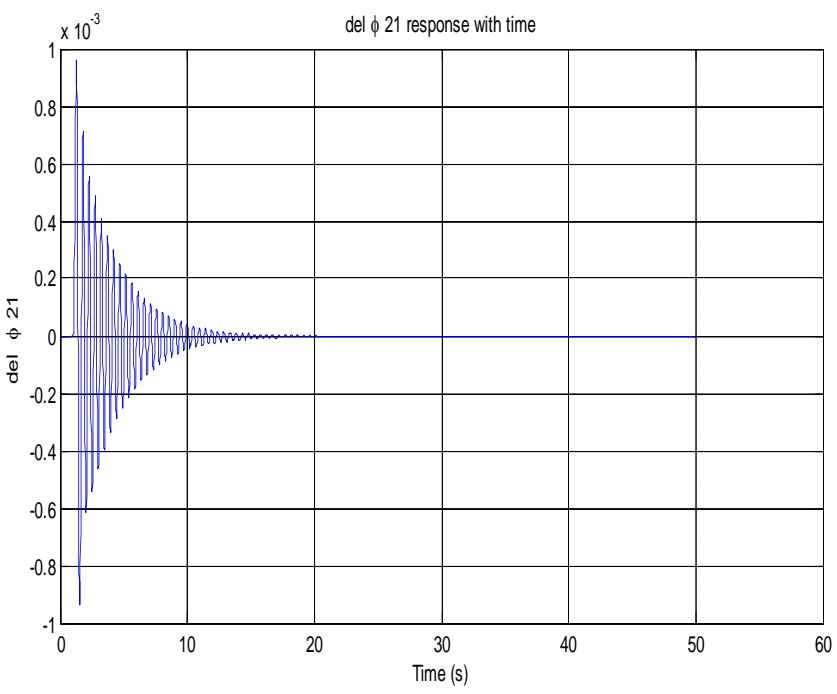

Fig-5.8 Response of $\Delta \Phi_{21}$ with time
It is clearly seen in figure 5.1 through 5.8 ; section $2,2.1$, that as soon as disturbance is created in the system comprising of facts device is now able to find new equilibrium state via presence of damping component as evident in phase portrait available. The same can also be inferred from different responses of state variables with time.

\section{CONCLUSIONS}

In this paper steady state analysis of a multi machine power system that is 3 machines and 9 bus power system about an initial operating condition available from load flow is analyzed. Multi machine power system is successfully analyzed with and without TCPS FACTS device based controller. The studies conducted in this paper yield the following conclusions:

1. It is observed that, test power system without controller is in purely oscillatory mode due to insufficient damping in the system.

2. By incorporating TCPS FACTS device based controller damping is introduced in system, damping of electromechanical mode of oscillation and steady state stability are enhanced by its usage.

3. The effectiveness of the control scheme for enhancement in damping has been successfully analyzed through eigen value analysis, phase portrait and time-domain simulations of linearized model for an operating condition.

\section{REFERENCES}

[1]. Divya Rishi Shrivastava, "Synchronizing and Damping Effects of TCPS and TCSC Based Coordinated Damping Controllers: Single Machine Infinite Bus", IJLTEMAS, Vol. III, Issue IX, September 2014.

[2]. K.R.Padiyar, "FACTS Controllers in Power Transmission and Distribution", New Age International Limited, Publishers, 2013

[3]. Fernando Javier De Marco, Nelson Martins, and Julio Cesar RezendeFerraz, "An Automatic Method for Power System Stabilizers Phase Compensation Design",IEEE Trans. On power systems, vol. 28, no. 2, may 2013.

[4]. P. Kundur, "Power System Stability and Control", Tata McGraw-Hill,2012.

[5]. M. A. Abido, "Power System Stability Enhancement Using Facts Controllers: A Review", The Arabian Journal for Science and Engineering, vol. 34, 2009.

[6]. "IEEE Guide for Synchronous Generator Modeling Practice and Application in Power System Stability Analysis", IEEE Power Engineering Society, Sponsor, April 2008.

[7]. IEEE/CIGRE Joint Task Force on Stability Terms and Definitions, "Definition and Classification of Power System Stability", IEEE TRANSACTIONS ON Power Systems, VOL. 19, NO. 2, May 2004.

[8]. Paul M Anderson, A.A .Fouad, “ Power System Control And Stability, ”Wiley,IEEE press, October 2002.

[9]. N. G. Hingorani, L. Gyugyi, "Understanding FACTS Concepts and Technology of Flexible AC Transmission Systems", IEEE Power Engineering Society, Sponsor,2000. 
[10]. Ramnarayan Patel, T. S. Bhatti and D. P. Kothari, "MATLAB/Simulink-based transient stability analysis of a multimachine power system", International Journal of Electrical Engineering Education 39/4.

[11]. "IEEE Guide for Synchronous Generator Modeling Practices in Stability Analyses", IEEE Power Engineering Society, Sponsor, March 21,1991.

[12]. Kai Xing, "Application of Thyristor-Controlled Phase shifters to minimize real power losses and augment stability of power systems",IEEE transaction on Energy Conversion ,vol. 3,No. 4,December 1988

[13]. IEEE Symposium on "Proposed Terms and Definitions For Power System stability", IEEE Tran. On power Apparatus and system.vol.PAS-101, no.7 July 1982

[14]. A. M. Liapunov, "The general problem of the stability of motion", 1892.

[15]. A. Chipperfield, P. Fleming, H. Pohlheim, C. Fonseca, "Genetic Algorithm TOOLBOX for Use with MATLAB", Mathworks.in.

[16]. NPTEL Online, "Power System Analysis", Module 4, IIT Kanpur, NPTEL.

Appendix I (generator data)

\begin{tabular}{|c|c|c|c|}
\hline Generator & 1 & 2 & 3 \\
\hline Rated MVA & 247.5 & 192 & 128 \\
\hline $\mathrm{kV}$ & 16.5 & 18 & 13.8 \\
\hline Power factor & 1.0 & 0.85 & 0.85 \\
\hline Type & Hydro & Steam & Steam \\
\hline Speed & $180 \mathrm{r} / \mathrm{min}$ & $3600 \mathrm{r} / \mathrm{min}$ & $3600 \mathrm{r} / \mathrm{min}$ \\
\hline$x_{d}$ & 0.1460 & 0.8958 & 1.3125 \\
\hline$x_{d}$ & 0.0608 & 0.1198 & 0.1813 \\
\hline$x_{q}$ & 0.0969 & 0.8645 & 1.2578 \\
\hline$x_{q}{ }^{\prime}$ & 0.0969 & 0.1969 & 0.25 \\
\hline$x_{2}$ (leakage) & 0.0336 & 0.0521 & 0.0742 \\
\hline$T_{d 0}=$ & 8.96 & 6.00 & 5.89 \\
\hline$T_{q 0}$ & 0 & 0.535 & 0.600 \\
\hline Stored energy & $2364 \mathrm{MW}$ & 640 MW.s & 301 MW.s \\
\hline at rated speed &.$s$ & & \\
\hline
\end{tabular}

Reactance values are in pu on a 100-MVA base

Appendix II. (Reduced Y Matrix)

$\left[\begin{array}{lll}0.8455-2.9883 \mathrm{i} & 0.2871+1.5129 \mathrm{i} & 0.2096+1.2256 \mathrm{i} \\ 0.2871+1.5129 \mathrm{i} & 0.4200-2.7239 \mathrm{i} & 0.2133+1.0879 \mathrm{i} \\ 0.2096+1.2256 \mathrm{i} & 0.2133+1.0879 \mathrm{i} & 0.2770-2.3681 \mathrm{i}\end{array}\right]$

Appendix III. (Synchronizing components, For Figure 1)

$\left[\begin{array}{ccc}0 & 2.0511 & 1.6139 \\ 1.8329 & 0 & 1.3016 \\ 1.5005 & 1.3641 & 0\end{array}\right]$

Appendix IV. (Controller parameters)

\begin{tabular}{|l|l|l|l|}
\hline $\mathrm{T} 1=0.017 \mathrm{~s}$ & $\mathrm{~T} 2=0.02 \mathrm{~s}$ & $T_{\mathrm{w}}=10 \mathrm{~s}$ & $\mathrm{~K}=0.1$ \\
\hline
\end{tabular}

Appendix V. (Synchronizing components, For Figure 3)

$\left[\begin{array}{ccc}0 & 1.6971 & 1.3357 \\ 1.5060 & 0 & 1.1281 \\ 1.2508 & 1.1803 & 0\end{array}\right]$

\section{BIOGRAPHIES}

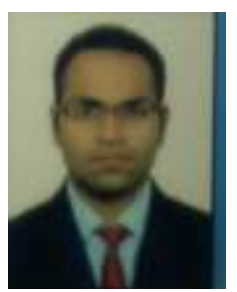

Divya Rishi Shrivastava (M'26) is Assistant Professor in Electrical \& Electronics Engineering Dept., Manipal University Jaipur, Rajasthan, India. He received M.Tech. in 2014 from NIT Silchar, India

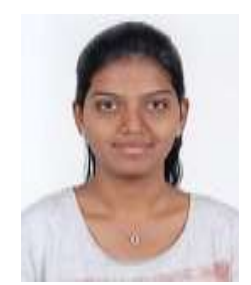

Daneshwari R Akkishetti (F'22) is lecturer in Electrical \& Electronics Engineering department, Jain College of Engineering Belgaum Karnataka, India. She received B.E. degree in 2015 from JCE, Belgaum, VTU, India. 